\title{
Ringer's lactate and red blood cells: is there sufficient evidence to recommend for routine use?
}

\author{
Elianna Saidenberg, MD · Alan Tinmouth, MD
}

Published online: 10 March 2009

(c) Canadian Anesthesiologists' Society 2009

Blood components are biological products that are collected from volunteer blood donors and stored for varying periods of time prior to being reinfused in patients. It is critically important to maintain the viability and functionality of the collected blood when the blood products are being stored and administered to ensure they will function flawlessly when transfused to patients. The process of blood collection has evolved significantly since blood cell preservation was first introduced by Rous and Tourner during World War I. The need for anticoagulation after collection is one unchanging feature that remains, and citrate has been used as the primary anticoagulant since the time of Rous and Turner. Once collected, a unit of whole blood is separated into its various components, i.e., red blood cells, plasma, and platelets, so that each component can be stored under optimal conditions. A glucose source,

E. Saidenberg, MD · A. Tinmouth, MD ( $\varangle)$

Department of Hematology and Transfusion Medicine,

The Ottawa Hospital and the University of Ottawa,

Ottawa, ON, Canada

e-mail: atinmouth@ottawahospital.on.ca

\section{A. Tinmouth, MD}

Department of Medicine, The Ottawa Hospital and the University of Ottawa, 501 Smyth Rd, Box 201, Ottawa, ON K1H 8L6, Canada

\author{
A. Tinmouth, MD \\ University of Ottawa Centre for Transfusion Research, \\ Ottawa, ON, Canada
}

A. Tinmouth, MD

Clinical Epidemiology Program, Ottawa Health Research

Institute, Ottawa, ON, Canada

\author{
A. Tinmouth, MD \\ Canadian Blood Services, Ottawa, ON, Canada
}

almost invariably dextrose, must be added to ensure preservation of red cells. Over time, other elements have been added to the preservative solutions to prolong the storage period of the red blood cells. In Canada, whole blood is currently collected into citric acid, sodium citrate, dextrose, sodium biphosphate (CPD or CP2D), or CPD with adenine (CPD-A1 or CPD-A2). After the plasma and platelets are removed from the red blood cells, a nutrient solution is added to the red blood cells. In Quebec, an additive solution 3 (AS-3), which contains additional citrate, is added to the CP2D red blood cells. Since the Canadian Blood Service's recent change to a buffy coat manufacturing process, the saline adenine glucose mannitol (SAGM) solution, which contains no additional citrate, is added to CPD red blood cells. The final packed red blood cell product (approximately $350 \mathrm{ml}$ ) is intended for transfusion at a rate of $2-5 \mathrm{ml} \cdot \mathrm{min}^{-1}$ without further manipulation.

In Canada, standards regarding the production and storage of blood products are established by Health Canada, and in the USA, they are established by the Food and Drug Administration (FDA). Different national standards for plasma and platelets include minimum levels of coagulation proteins, and platelet function, recovery and survival studies. The standards in Europe and North America regarding red blood cells specify 24 -h survival of more than $70 \%$ of cells, a minimum level of hemoglobin content, and/or a maximum level of hemolysis. The administration of blood products is within the scope of clinical practice, similar to giving drugs for off-label indications and, as such, is not covered by federal regulations. However, the quality of blood products can be significantly compromised during administration. The potential consequences of administering red blood cells with other solutions or medications are lysis of the red blood cells and the formation of red blood cell clots. Both 
of these events will diminish the effectiveness of the red blood cell transfusion and could result in potentially serious adverse consequences for the patient. In North America, the AABB (formerly the American Association of Blood Banks), the Canadian Standards Association, and the Canadian Society of Transfusion Medicine have standards ${ }^{1-3}$ pertaining to the administration of blood products to ensure that the function of the blood products is maintained and the risk of adverse consequences is minimized. These standards stipulate that no medications or solutions, other than normal saline, shall be added to blood components during administration, unless they are approved by the FDA/Health Canada, or unless there is documentation to demonstrate that the blood component is not adversely affected. $^{1-3}$ Guidelines issued by various organizations, such as the Canadian Blood Services or hospital-based transfusion medicine services will usually reflect these standards. However, these guidelines and recommendations against infusing specific substances together with red blood cells are not necessarily based on evidence indicating that these substances pose a danger; rather, they are based on the lack of data supporting their safety. Indeed, there is very little evidence to support the safety in administering specific medications or alternate intravenous solutions with red blood cells. The exceptions are certain narcotics given via patient-controlled analgesia pumps (morphine, hydromorphone, and ketamine) and specific intravenous solutions, such as normosol-R $\mathrm{pH} 7.4$ and plasma-lyte-A injection $\mathrm{pH} 7.4 .^{4}$

In most clinical situations, it is not problematic to use a dedicated intravenous line and to prime with normal saline for the administration of red blood cells. However, there are certain situations where solutions other than saline may be indicated or where multiple intravenous solutions and medications need to be administered simultaneously. Compared to normal saline, Ringer's lactate (RL) will not cause a hyperchloremic acidosis during large volume infusions. Therefore, it is the preferred fluid for resuscitation and is commonly administered with red blood cell transfusions. In this issue of the Journal, Albert et al. ${ }^{5}$ present evidence and argue that RL can safely be used to dilute packed red blood cells when rapid transfusion is required. The primary concern with infusing $\mathrm{RL}$ with red blood cells is calcium neutralizing the citrate and forming clots. Based on a macroscopic evaluation of microaggregate $(40 \mu \mathrm{l})$ blood filters and biochemical markers of coagulation when AS-3 red blood cells were diluted up to tenfold with RL, Albert et al. found no evidence of increased clot formation in this rapid transfusion model.

The study raises several questions for practitioners in the field of transfusion medicine. First, we may try to recall the reasons for RL being considered unsafe in the first place. Next, we may be concerned about the type and amount of evidence that regulating bodies require when considering a product safe or unsafe for such an indication. Since a variety of blood products may be used in massive transfusion situations that necessitate rapid transfusion, we may also question whether compatibility with all transfusable components should be proven or whether assurance should only be required for compatibility with red blood cells.

In 1975, Ryden and Oberman demonstrated incompatibility between red blood cells preserved in a CPD anticoagulant and RL. ${ }^{6}$ The calcium content of RL is capable of overcoming the chelating effect of citrate. Evidence of clot formation was observed in incubated samples of RL and red blood cells and in the intravenous tubing at flow rates mimicking routine transfusions. It is feared that the resultant clots could give rise to microaggregates entering the circulation. While the clinical relevance of these transfused microaggregates is not clear, there is a concern that they may contribute to respiratory compromise or some other organ dysfunction. ${ }^{7}$ The study by Albert et $a l{ }^{5}$ and a previous trial by King et al. ${ }^{8}$ did not show evidence of increased clot formation in $\mathrm{AS}-3^{5}$ or CPDA $-1^{8}$ red blood cells in rapid transfusion models. Importantly, these studies looked for confirmation of microaggregates by examining $40 \mu \mathrm{l}$ filters and biochemical evidence of fibrin clot formation. An additional study also examined CPD red blood cells in a transfusion model; however, it only looked for evidence of clots in $170 \mu \mathrm{l}$ filters which would not be expected to capture microaggregates. ${ }^{9}$

The weight of evidence would suggest that adding RL to red blood cell concentrates could potentially cause problems with clotting, although this risk would appear to be mitigated with rapid infusions. However, as the clinical consequence of transfusing small clots or microaggregates is not known and microaggregate filters are not routinely used for transfusion of allogeneic red cell concentrates, normal saline should remain the standard solution for routine transfusion of red blood cells. Generally, there is no reason to dilute red blood cell concentrates with $\mathrm{RL}$ or to use this as a priming solution, and these practices should be avoided whenever possible. Given the advantages of resuscitation with $\mathrm{RL}$ over normal saline, running red blood cells and RL through the same line may be unavoidable. The current study supports the premise that, in specific situations, the beneficial effects of the RL may outweigh the disadvantages of combining RL with red blood cells. However, the evidence would only support the use of RL in rapid transfusion settings encountered in the operating theater and trauma settings. Ideally, there should be additional data from animal and human studies to further support this practice in these limited situations. These studies should also be extended to include SAGM red blood cell concentrates. 
Given the limited number of laboratory studies, the lack of in vivo data, and the concern for adverse events associated with transfusing red blood cells and RL, transfusion guidelines cannot, and should not, suggest that RL is safe to administer with red blood cells. In specific circumstances, the risk to benefit assessment may favor transfusing red blood cells with RL; however, this would be the exception. This exceptional use of $\mathrm{RL}$ with red blood cells could be considered in guidelines for cases with limited venous access, such as those that approximate the co-administration of narcotics and red blood cells. ${ }^{4}$

This editorial underscores the importance of crosscommunication among the various disciplines of medicine that must work together to ensure improved outcomes for patients needing blood transfusions. The authors of this editorial and our colleagues in transfusion medicine and blood banking continue to work to improve the safety and efficacy of blood donation, storage, and transfusion. However, we recognize that advancing the practices of transfusion medicine requires collaboration with our colleagues outside the blood bank. Hopefully, such collaboration will facilitate a better understanding of the variables that impact on patient safety and outcomes and only come into play once the blood is hanging for infusion. With any luck, continued investigation to answer the remaining questions about the compatibility of RL with blood components will be made possible with ongoing dialog between specialties.

\section{Le lactate ringer et les érythrocytes: les données probantes sont-elles suffisantes pour recommander un usage de routine?}

Les composants sanguins sont des produits biologiques recueillis de donneurs volontaires et stockés pour des périodes plus ou moins longues avant d'être transfusés à des patients. Il est essentiel de maintenir la viabilité et la fonctionnalité du sang récolté lorsque les produits sanguins sont stockés et administrés afin de garantir qu'ils fonctionneront sans aucun problème quand ils seront transfusés à des patients. Le processus de collecte du sang a considérablement évolué depuis la première introduction d'une méthode de préservation des globules rouges par Rous et Tourner pendant la Première Guerre mondiale. La nécessité d'une méthode d'anticoagulation après la collecte est une des caractéristiques qui n'a pas changé et demeure. Le citrate est l'anticoagulant par excellence depuis Rous et Tourner. Une fois collectée, une unité de sang total est divisée en ses composants divers, soit les érythrocytes, le plasma et les plaquettes, afin que chaque composant puisse être stocké dans des conditions optimales. Une source de glucose, la plupart du temps le dextrose, doit être ajoutée afin de garantir la préservation des érythrocytes. Au fil du temps, d'autres éléments ont été ajoutés aux solutions de préservation afin de prolonger la période de stockage des érythrocytes. Au Canada, le sang total est présentement collecté dans l'acide citrique, le citrate de sodium, le dextrose, le diphosphate de sodium (CPD ou CP2D), ou le CPD avec adénine (CPD-A1 ou CPD-A2). Une fois que le plasma et les plaquettes sont séparés des érythrocytes, une solution nutritive est ajoutée aux érythrocytes. Au Québec, une solution additive 3 (AS-3) contenant davantage de citrate est ajoutée aux érythrocytes CP2D. Depuis que la Société canadienne du sang a changé son processus de fabrication pour y inclure la centrifugation leucocyto-plaquettaire, une solution saline d'adénine-glucose-mannitol (SAGM), qui ne contient pas de citrate supplémentaire, est ajoutée aux érythrocytes CPD. Le concentré de globules rouges final (environ $350 \mathrm{ml}$ ) est destiné à être transfusé à un taux de $2-5 \mathrm{ml} \cdot \mathrm{min}^{-1}$ sans autre manipulation.

$\mathrm{Au}$ Canada, les normes concernant la production et le stockage des produits sanguins sont établies par Santé Canada; aux États-Unis, la Food and Drug Administration (FDA) s'en charge. Parmi les normes nationales qui diffèrent pour le plasma et les plaquettes, citons les niveaux minimaux de protéines de coagulation, et les études portant sur la fonction, la récupération et la survie des plaquettes. Les normes européennes et nord-américaines concernant les érythrocytes spécifient une survie de $24 \mathrm{~h}$ pour plus de $70 \%$ des érythrocytes, un niveau minimal de contenu d'hémoglobine, et/ou un niveau maximal d'hémolyse. L'administration des produits sanguins tombe sous la coupe de la pratique clinique, tout comme l'administration de médicaments pour des indications non conformes ; par conséquent, leur administration n'est pas couverte par les règlements fédéraux. Toutefois, la qualité des produits sanguins peut être considérablement compromise pendant leur administration. Les conséquences potentielles d'une administration d'érythrocytes avec d'autres solutions ou médicaments incluent une lyse des érythrocytes et la formation de caillots d'érythrocytes. Ces deux événements diminueront l'efficacité de la transfusion d'érythrocytes et pourraient provoquer des effets secondaires potentiellement graves pour les patients. En Amérique du Nord, l'AABB (anciennement l'American Association of Blood Banks), l'Association canadienne de normalisation et la Société canadienne de médecine transfusionnelle disposent de normes ${ }^{1-3}$ concernant l'administration de produits sanguins afin de garantir que la fonction des produits sanguins est maintenue et que le risque de conséquences néfastes est minimisé. Ces normes stipulent qu'aucun médicament ou 
solution autre qu'une solution salée isotonique ne sera ajoutée aux composants sanguins pendant l'administration, à moins qu'ils ne soient approuvés par la FDA/Santé Canada, ou à moins qu'il existe des données probantes indiquant que le composant sanguin n'est pas affecté de façon néfaste. ${ }^{1-3}$ Les directives publiées par diverses organisations, comme la Société canadienne du sang ou les services de médecine transfusionnelle dans les hôpitaux, refléteront en général ces directives. Cependant, ces directives et recommandations contre la transfusion de substances spécifiques conjointement à l'administration d'érythrocytes ne sont pas forcément fondées sur des données probantes démontrant que ces substances posent un risque; en fait, elles sont plutôt fondées sur le manque de données soutenant leur innocuité. En effet, il n'existe que très peu de données probantes appuyant l'innocuité de l'administration de médicaments spécifiques ou de solutions intraveineuses alternatives avec les érythrocytes. Certains morphiniques administrés par des pompes d'analgésie contrôlées par le patient (morphine, hydromorphone, kétamine) et certaines solutions intraveineuses spécifiques, telles que le normosol-R pH 7,4 et l'injection de plasmalyte A pH 7,4 sont des exceptions. ${ }^{4}$

Dans la plupart des situations cliniques, le fait d'utiliser une voie intraveineuse dédiée et d'amorcer cette dernière avec une solution salée isotonique pour l'administration d'érythrocytes ne pose pas de problème. Dans certaines situations toutefois, il se peut que des solutions autres que la solution salée isotonique soient indiquées ou que plusieurs solutions et médicaments intraveineux doivent être administrés simultanément. Par rapport à une solution salée isotonique, le lactate Ringer (LR) ne provoquera pas d'acidose hyperchlorémique pendant des perfusions de volume important. Pour cette raison, le LR constitue le liquide de choix pour la réanimation et il est fréquemment administré avec des transfusions d'érythrocytes. Dans ce numéro du Journal, Albert et coll. ${ }^{5}$ présentent des données et soutiennent que le LR peut être utilisé en toute sécurité pour diluer les concentrés de globules rouges lorsqu'une transfusion rapide est nécessaire. La préoccupation première lorsqu' on perfuse du LR avec des érythrocytes est le fait que le calcium neutralise le citrate et forme des caillots. En se fondant sur une évaluation macroscopique de la présence de microagrégats $(40 \mu \mathrm{l})$ au niveau des filtres sanguins et des marqueurs biochimiques de coagulation lorsque les érythrocytes AS-3 étaient dilués jusqu'à dix fois dans le LR, Albert et coll. n'ont pas trouvé d'augmentation de la formation de caillots dans ce modèle de transfusion rapide.

Cette étude soulève plusieurs questions pour les praticiens travaillant dans le domaine de la médecine transfusionnelle. En premier lieu, il faut nous rappeler les raisons pour lesquelles le LR est considéré a priori comme non sécuritaire. Ensuite, nous pourrions être inquiets concernant le type et la quantité de données probantes que les organismes de réglementation requièrent lorsqu'ils examinent si un produit est sécuritaire ou non pour une telle indication. Étant donné que toutes sortes de produits sanguins peuvent être utilisés dans les situations de transfusions massives nécessitant une transfusion rapide, nous pourrions également soulever la question de savoir si la compatibilité devrait être prouvée pour tous les composants transfusables ou si l'assurance d'une compatibilité avec les érythrocytes suffit.

En 1975, Ryden et Oberman ont démontré une incompatibilité entre les érythrocytes préservés dans un anticoagulant CPD et le $\mathrm{LR}^{6}{ }^{6}$ Le contenu calcique du LR est capable de supplanter l'effet de chélation du citrate. Des observations suggérant la formation de caillots ont été faites dans des échantillons incubés de LR et d'érythrocytes et dans les voies intraveineuses à des débits imitant les transfusions de routine. Dès lors, il est à craindre que les caillots ainsi formés pourraient provoquer des microagrégats qui s'introduiraient dans la circulation. Bien que la pertinence clinique de ces microagrégats transfusés ne soit pas claire, la crainte est qu'ils contribuent aux complications respiratoires ou à une autre dysfonction des organes. ${ }^{7}$ L'étude d'Albert et coll. ${ }^{7}$ ainsi qu'une étude précédente de King et coll. ${ }^{8}$ n'ont pas mis en évidence d'augmentation de la formation de caillots dans les érythrocytes AS- $3^{5}$ ou CPDA $-1^{8}$ dans des modèles de transfusion rapide. Il faut souligner que ces études ont cherché la confirmation de microagrégats dans des filtres de $40 \mu \mathrm{l}$ et des données biochimiques de formation de caillots de fibrine. Une étude supplémentaire a également examiné des érythrocytes CPD dans un modèle de transfusion; cependant, cette étude n'a recherché la présence de caillots que dans des filtres de $170 \mu \mathrm{l}$, dans lesquels les microagrégats pourraient ne pas être capturés. ${ }^{9}$

La quantité de données probantes à notre disposition suggère que l'ajout de LR à des concentrés d'érythrocytes pourrait potentiellement causer des problèmes de formation de caillots, bien que ce risque semble être neutralisé par des transfusions rapides. Toutefois, étant donné que nous ne connaissons pas la conséquence clinique d'une transfusion de petits caillots ou de microagrégats et que les filtres à microagrégats ne sont pas utilisés dans la pratique de routine pour les transfusions de concentrés d'érythrocytes allogènes, la solution salée isotonique devrait dès lors demeurer la norme pour la transfusion de routine d'érythrocytes. En règle générale, il n'y a aucune raison de diluer les concentrés d'érythrocytes avec du LR ou de l'utiliser comme solution d'amorçage, et ces pratiques devraient être évitées autant que possible. Étant donné les avantages de la réanimation avec le LR comparativement à une solution salée isotonique, il se peut qu'il soit inévitable 
d'avoir une seule voie pour les érythrocytes et le LR. L'étude en question ici soutient l'hypothèse que, dans certaines situations spécifiques, les effets bénéfiques du LR pourraient surpasser les inconvénients de sa combinaison avec les érythrocytes. Néanmoins, les données présentées ici n'appuieraient l'utilisation du LR que dans des contextes de transfusion rapide rencontrés dans la salle d'opération et les contextes de trauma. Nous devrions, dans l'idéal, disposer de données supplémentaires tirées d'études animales et humaines afin d'appuyer plus avant cette pratique dans ces situations limitées. Ces études devraient également inclure les concentrés d'érythrocytes SAGM.

Étant donné le nombre limité d'études de laboratoire, le manque de données in vivo, et les préoccupations concernant la possibilité d'événements néfastes associés à une transfusion d'érythrocytes avec du LR, les directives de transfusion ne peuvent pas, et ne devraient pas, suggérer que le LR est sécuritaire pour une administration conjointe aux érythrocytes. Dans certaines circonstances spécifiques, l'évaluation des risques et bienfaits pourrait justifier une transfusion d'érythrocytes avec du LR; cependant, il s'agirait là de l'exception. Cette utilisation exceptionnelle du LR avec des érythrocytes pourrait être envisagée dans les directives pour les cas où l'accès veineux est limité, tels que les cas qui ressemblent à ceux où la co-administration de morphiniques et d'érythrocytes est requise. ${ }^{4}$

Cet éditorial souligne l'importance de la communication entre les diverses disciplines médicales qui doivent collaborer afin de garantir de meilleurs devenirs aux patients nécessitant des transfusions sanguines. Les auteurs de cet éditorial et nos collègues en médecine transfusionnelle et dans les banques de sang continuent de travailler afin d'améliorer la sécurité et l'efficacité des dons de sang, du stockage et de la transfusion. Cependant, nous sommes conscients que pour faire progresser les pratiques de la médecine transfusionnelle, il nous faut collaborer avec nos collègues qui ne travaillent pas dans la banque de sang.
Nous espérons qu'une telle collaboration facilitera une meilleure compréhension des variables qui ont un impact sur la sécurité et les devenirs de patients, variables qui ne commencent à jouer un rôle que lorsque la perfusion est prête à être administrée. Avec un peu de chance, des recherches continues afin de répondre aux questions encore sans réponse concernant la compatibilité du LR avec les composants sanguins verront le jour grâce au dialogue permanent entre les diverses spécialités.

Conflicts of interest Elianna Saidenberg, a transfusion medicine fellow at the Ottawa Hospital, is sponsored by the Canadian Blood Services. Alan Tinmouth is a medical consultant with the Canadian Blood Services and has received research grants from the Canadian Blood Services.

\section{References}

1. $A A B B$. Standards for blood banks and transfusion services. 25th ed. Bethesda, Maryland: AABB; 2008.

2. Canadian Standards Association. Blood and blood components. Mississauga: Canadian Standards Association; 2004.

3. Canadian Society for Transfusion Medicine. CSTM standards for hospital transfusion services. 2nd ed. Toronto, Canada: Canadian Society for Transfusion Medicine; 2009.

4. Sink B. Administration of blood components. In: Roback J, Combs M, Grossman B, Hillyer C, editors. AABB technical manual. Bethesda: American Association of Blood Banks; 2008. p. 613-24.

5. Albert K, van Vlymen J, James P, Parlow J. Ringer's lactate is compatible with the rapid infusion of AS-3 preserved packed red blood cells. Can J Anesth 2009; 56: 5.

6. Ryden SE, Oberman HA. Compatibility of common intravenous solutions with CPD blood. Transfusion 1975; 15: 250-5.

7. Klein G, Anstee D. Some unfavourable effects of transfusion. In: Huxley R, Khan M, Charman K, editors. Mollison's blood transfusion in clinical medicine. Malden: Blackwell Publishing; 2005. p. 666-700.

8. King WH, Patten ED, Bee DE. An in vitro evaluation of ionized calcium levels and clotting in red blood cells diluted with lactated Ringer's solution. Anesthesiology 1988; 68: 115-21.

9. Lorenzo M, Davis JW, Negin S, Kaups K, Parks S, Brubaker D, et al. Can Ringer's lactate be used safely with blood transfusions? Am J Surg 1998; 175: 308-10. 